\title{
Sobrepeso, obesidade e hipertensão arterial sistêmica em idosos: uma revisão de literatura
}

Overweight, obesity and systemic arterial hypertension in the elderly: a literature review

Raquel dos Santos ${ }^{1}$; Rafael Santos Barbosa ${ }^{2}$; Yasmin Assis Lozado ${ }^{2}$; Sabrina da Silva Caires ${ }^{2 *}$; Lucas dos Santos ${ }^{2}$

${ }^{1}$ Faculdade Maria Milza - FAMAM, Governador Mangabeira - Bahia, Brasil, 44350-000, r.nutrisb@gmail.com

${ }^{2}$ Universidade Estadual do Sudoeste da Bahia - UESB. rafabarbosa16rb@gmail.com; yasmin751957@gmail.com; sabrinacaires9@hotmail.com (autor correspondente);

lsantos.ed.f@gmail.com

\section{Resumo}

O envelhecimento é composto por alterações morfológicas, a exemplo do acúmulo excessivo de gordura, que torna o idoso mais propenso ao acometimento de comorbidades, como a Hipertensão Arterial Sistêmica (HAS), que, por sua vez, proporciona maior risco para desfechos desfavoráveis à saúde. Assim, o presente estudo objetiva averiguar a associação entre sobrepeso/obesidade e HAS em idosos. Trata-se de uma revisão de literatura integrativa, realizada nas bases de dados Latina- Americana e do Caribe em Ciências da Saúde e Scientific Electronic Library Online. Verificou-se 470 manuscritos científicos. Deste, quatro foram selecionados à revisão. As populações estudadas variaram de 477 a 963 idosos, com média de idade entre $65,0 \pm 9,0$ e 73,0 $\pm 8,9$ anos. As prevalências de sobrepeso/obesidade oscilaram entre 34,2 e 77,4\%, enquanto as de hipertensão arterial, de 62,8 a 76,8\%. Observou-se, em um dos estudos, que os idosos obesos apresentaram 3,85 vezes mais chances de serem hipertensos, quando comparados aos eutróficos. Outro estudo mostrou que as idosas e os idosos hipertensos, possuíam, respectivamente, em média, um índice de massa corporal 3,22 e 2,27 kg/m² maior que os não hipertensos. Ademais, observou-se entre os idosos obesos, uma prevalência de hipertensão $40 \%$ maior que a entre os com peso normal. Averiguou-se elevadas prevalências de hipertensão arterial e sobrepeso/obesidade, bem como associações entre estas condições nos idosos avaliados. Assim, torna-se imprescindível a adoção de ações de vigilância à saúde, como o monitoramento do estado nutricional, a fim de se triar os idosos com maior vulnerabilidade à HAS e suas possíveis complicações.

Palavras-chave: Envelhecimento. Estado Nutricional. Pressão Arterial Elevada. 


\begin{abstract}
Aging is composed by morphological changes, such as excessive fat accumulation, wich makes the elderly more prone to comorbities development, as Systemic Arterial Hypertension (SAH), that provides a greater risk for unfavorable health outcomes. Thus, the present study aimed to investigate the association between overweight/obesity and SAH in the elderly. This is an integrative literature review, made in the Latin American and Caribbean databases on Health Sciences and Scientific Electronic Library Online. There were 470 scientific manuscripts. Wich four of then selected for review. The studied populations ranged from 477 to 963 elderly people, with a avegare age between $65.0 \pm 9.0$ and $73.0 \pm 8.9$ years. The overweight/obesity prevalence ranged from 34.2 to $77.4 \%$, while that of arterial hypertension, from 62.8 to $76.8 \%$. It was observed, in one of the studies, that obese elderly were 3.85 times more likely to be hypertensive, when compared to eutrophic individuals. Another study showed that elderly women and elderly people with hypertension had, respectively, an average body mass index of 3.22 and $2.27 \mathrm{~kg} / \mathrm{m}^{2}$ higher than non-hypertensive individuals. Furthermore, there was a prevalence of hypertension $40 \%$ higher among obese elderly people than those with normal weight. There was a high prevalence of arterial hypertension and overweight/obesity, as well as associations between these conditions in the elderly evaluated. Thus, it's essential to adopt health surveillance actions, such as monitoring nutritional status, in order to screen elderly people who are more vulnerable to SAH and its possible complications.
\end{abstract}

Keywords: Aging. Nutritional Status. High Blood Pressure.

\title{
1. Introdução
}

O envelhecimento é composto por alterações endócrinas, a exemplo de diminuições nos níveis dos estrogênios nas mulheres (KARVONEN-GUTIERREZ; KIM, 2016) e menor produção e circulação da testosterona nos homens (CALIXTO; PRAZERES, 2019), que propiciam agravos à condição nutricional, como aumentos na disposição da gordura corporal (JAFARINASABIAN et al., 2017).

O elevado acúmulo do tecido adiposo tem sido evidenciado, na literatura, como uma importante doença crônica, que, por sua vez, torna os idosos mais vulneráveis ao acometimento de comorbidades, como a Hipertensão Arterial Sistêmica (HAS) (MALACHIAS et al., 2016).

Diante disto, tem sido verificado que na população brasileira, a ocorrência da HAS tende a aumentar linearmente ao avanço da idade dos indivíduos, apresentando prevalências 
estimadas de $49,5 \%$ e $60,9 \%$ nos grupos etários com idade entre 55 a 64 anos e 65 anos ou mais, respectivamente (BRASIL, 2019).

As elevadas prevalências de HAS, nos indivíduos mais velhos, configuram um importante problema de saúde pública, posto que o alto nível de pressão arterial, quando sustentado, representa maior probabilidade para infarto agudo do miocárdio, acidente vascular encefálico e doenças renais crônicas, além de ser um considerável fator de risco à mortalidade (MALACHIAS et al., 2016).

Assim, torna-se necessário a identificação e reunião de evidências científicas, que retratem a relação entre excesso de peso, obesidade e HAS em pessoas idosas, para que se possa identificar os indivíduos com maior vulnerabilidade para desfechos adversos, proporcionando, assim, importantes subsídios às ações que visem o cuidado, promoção, atenção e preservação das condições de saúde desta população. Portanto, este estudo tem como objetivo averiguar a associação entre sobrepeso, obesidade e hipertensão arterial sistêmica em idosos.

\section{Material e Métodos}

Trata-se de uma revisão de literatura, na modalidade integrativa, construída a partir das seguintes etapas metodológicas: 1) definição do tema e formulação da questão norteadora, 2) escolha das bases de dados utilizadas na pesquisa, 3) estabelecimento dos critérios de inclusão e exclusão, 4) definição dos descritores, 5) pré-seleção dos artigos, 6) avaliação dos estudos pré-selecionados e seleção dos estudos incluídos na revisão, 7) interpretação dos resultados e 8) apresentação da revisão integrativa (SOUZA; SILVA; CARVALHO, 2010).

A coleta de dados foi realizada em setembro de 2019, para responder a seguinte questão norteadora: "quais as evidências que retratam a associação entre as condições de sobrepeso, obesidade e hipertensão arterial sistêmica em idosos?". Portanto, foram realizadas buscas dentro das bases de dados Literatura Latina- Americana e do Caribe em Ciências da Saúde e Scientific Electronic Library Online, utilizando a seguinte estratégia de busca: "hypertension" AND “nutritional status” AND “aging” OR “elderly” OR “older adults”.

Os critérios de inclusão foram: estudos originais, publicados entre 2013 e 2019, em 
língua portuguesa ou inglesa, disponíveis em texto completo, relacionados ao objetivo proposto. Todavia, foram adotados os seguintes critérios de exclusão: projetos de pesquisa, dissertações, monografias, teses, publicações em anais de eventos, cartas ao editor, artigos de revisão, artigos não disponíveis em formato completo ou duplicados.

A seleção dos estudos foi feita por dois revisores de modo simultâneo e independente. Contudo, na existência de dúvidas um terceiro revisor experiente foi consultado. A pesquisa foi realizada em quatro etapas: análise das duplicatas; leitura dos títulos e resumos; leitura dos artigos na íntegra e extração das principais informações. Os dados coletados dos estudos selecionados foram: autores, ano de publicação, local de realização e tipo do estudo, participantes (idade, sexo), critério utilizados para avaliação do estado nutricional e resultados.

Para avaliação dos estudos selecionados, foi utilizado a Classificação Hierárquica das Evidências para Avaliação dos Estudos, que contempla os seguintes níveis: 1) revisão sistemática ou metanálise; 2) ensaios clínicos randomizados; 3) ensaio clínico sem randomização; 4) estudos de coorte e de caso-controle; 5) revisão sistemática de estudos descritivos e qualitativos; 6) estudo transversal, descritivo ou qualitativo; 7) opinião de autoridades e/ou relatório de comitês de especialidades (MELNYK et al., 2010).

\section{Resultados}

Após as buscas, foram encontrados 470 artigos científicos, 270 na base de dados Literatura Latina - Americana e do Caribe em Ciências da Saúde e 200 no Scientific Electronic Library Online.

Foram aplicados os critérios de inclusão e exclusão após triagem dos estudos por títulos e resumos. Sendo assim, 20 artigos foram excluídos por estarem duplicados nas duas bases de dados, 65 por se tratarem de monografias, dissertações e teses, cinco por serem estudos de revisão e 365 por não estarem relacionados ao tema proposto.

Desta maneira, 15 artigos foram selecionados à leitura completa. Destes, quatro foram selecionados à revisão integrativa por atenderem ao objetivo e critérios propostos. 
Quanto aos aspectos metodológicos, os quatro estudos selecionados apresentaram delineamento transversal, possuindo, portanto, nível de evidência seis.

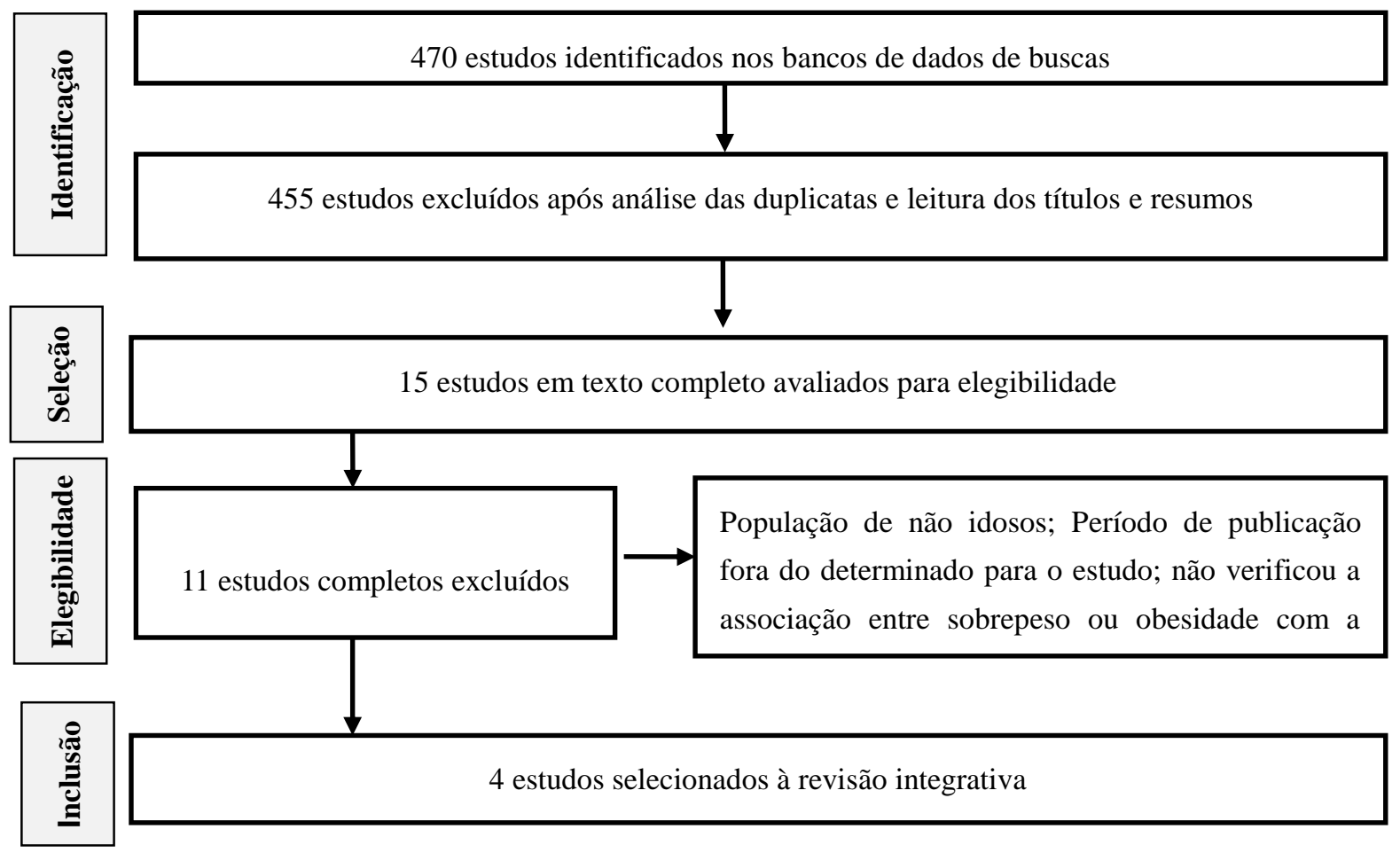

Figura 1. Fluxograma do processo de inclusão e exclusão dos artigos revisados. Jequié-BA, Brasil, 2020.

O quadro 1 apresenta a síntese dos artigos selecionados à revisão. As populações avaliadas variaram de 477 a 963 idosos, de ambos os sexos, com média de idade entre 65,0 \pm 9,0 e 73,0 $\pm 8,9$ anos. As prevalências de sobrepeso/obesidade, averiguadas, oscilaram entre 34,2 e 77,4\%, enquanto as de hipertensão arterial sistêmica variaram de 62,8 a 76,8\%.

Verificou-se em um dos estudos que os idosos obesos apresentaram 3,85 vezes mais chances de serem hipertensos, quando comparados aos eutróficos. Em outro estudo, observou-se que as idosas e os idosos hipertensos apresentaram, respectivamente, em média, um Índice de Massa Corporal (IMC) 3,22 e 2,27 kg/m maior que os avaliados sem hipertensão. Ademais, foi observado que idosos com obesidade apresentaram uma prevalência de hipertensão $40 \%$ maior quando comparados aos com peso normal $(p<0,05)$. 
Quadro 1. Principais características dos estudos selecionados à revisão integrativa. Jequié-BA, Brasil, 2019.

\begin{tabular}{|c|c|c|c|c|}
\hline $\begin{array}{c}\text { Autores } \\
\text { (Local/Ano) }\end{array}$ & Participantes & Idade & Critério EN & Resultados \\
\hline $\begin{array}{c}\text { Tavares et al. } \\
\text { (Uberaba-MG/ } \\
\text { 2018) }\end{array}$ & $\begin{array}{c}562 \text { idosos } \\
(\mathrm{F} \text { e } \mathrm{M})\end{array}$ & $\geq 60$ & Lipschitz (1994) & $\begin{array}{l}\text { Verificou-se prevalência de } 34,30 \% \text { de } \\
\text { sobrepeso/obesidade e que } 66,10 \% \text { dos } \\
\text { avaliados tinham HAS. Os idosos com } \\
\text { sobrepeso/obesidade apresentaram } 2,52 \text { vezes } \\
\text { mais chances para desenvolver a HAS } \\
(p<0,05) \text {. }\end{array}$ \\
\hline $\begin{array}{c}\text { Turi et al. } \\
\text { (Bauru-SP/20 } \\
\text { 13) }\end{array}$ & $\begin{array}{c}963 \text { idosos } \\
(\mathrm{F} \text { e } \mathrm{M})\end{array}$ & $\begin{array}{c}65,00 \\
\pm \\
9,00\end{array}$ & $\begin{array}{c}\text { Organização } \\
\text { Mundial de Saúde } \\
\text { (1998) }\end{array}$ & $\begin{array}{l}\text { Observou-se que } 22,4 \% \text { dos avaliados tinham } \\
\text { sobrepeso, } 40,6 \% \text { eram obesos e } 76,8 \% \\
\text { possuíam HAS. Os idosos com sobrepeso e } \\
\text { os com obesidade apresentaram, } \\
\text { respectivamente, } 1,2 \text { e } 1,4 \text { maior prevalência } \\
\text { de HAS quando comparados aos eutróficos } \\
(p<0,05) \text {. }\end{array}$ \\
\hline $\begin{array}{l}\text { Ferreira, } \\
\text { Monteiro e } \\
\text { Simões } \\
\text { (Rio de } \\
\text { Janeiro-RJ/20 } \\
\text { 18) }\end{array}$ & $\begin{array}{c}720 \text { idosos } \\
(\mathrm{F} \text { e } \mathrm{M})\end{array}$ & $\geq 60$ & $\begin{array}{c}\text { Organização } \\
\text { Mundial de Saúde } \\
\text { (1998) }\end{array}$ & $\begin{array}{l}\text { Entre os avaliados, } 62,8 \% \text { apresentaram HAS, } \\
57,5 \% \text { excesso de peso e } 19,9 \% \text { obesidade. Os } \\
\text { idosos com sobrepeso e os com obesidade } \\
\text { apresentaram } 1,7 \text { e } 3,85 \text { vezes mais chances, } \\
\text { respectivamente, para HAS, quando } \\
\text { comparados aos com peso adequado ( } p<0,05) \text {. }\end{array}$ \\
\hline $\begin{array}{l}\text { Leal Neto, } \\
\text { Barbosa e } \\
\text { Meneghini } \\
\text { (Antônio } \\
\text { Carlos-SC/201 } \\
\text { 6) }\end{array}$ & $\begin{array}{c}477 \text { idosos } \\
(\text { F e M) }\end{array}$ & $\begin{array}{l}73,00 \\
\pm \\
8,90\end{array}$ & $\begin{array}{l}\text { IMC avaliado } \\
\text { como variável } \\
\text { quantitativa } \\
\text { (contínua) }\end{array}$ & $\begin{array}{l}\text { Averiguou-se que as idosas e os idosos } \\
\text { hipertensos apresentaram, respectivamente, } \\
\text { em média, um IMC } 3,22 \text { e } 2,27 \mathrm{~kg} / \mathrm{m}^{2} \text {, maior } \\
\text { que os avaliados sem hipertensão }(p<0,05) \text {. }\end{array}$ \\
\hline
\end{tabular}

F: sexo feminino. M: sexo masculino. HAS: hipertensão arterial sistêmica. EN: estado nutricional. IMC: índice de massa corporal. $\mathrm{kg} / \mathrm{m}^{2}$ : quilograma por metro quadrado.

\section{Discussão}

A obesidade é uma doença crônica que tem apresentando-se como um problema de saúde pública mundial, posto os consideráveis aumentos observados em suas ocorrências, tanto em países desenvolvidos, quanto nos em desenvolvimento, afetando diferentes populações e faixas etárias (MARIE et al., 2014).

Por ser multifatorial esta morbidade pode apresentar diferentes etiologias, a exemplo dos 
aspectos genéticos, ambientais e psicossociais, além do alto consumo calórico, baixos níveis de atividade física e a adoção de elevado tempo despendido em comportamentos sedentários (SUZANNE; WRIGHT; ARONNE, 2012; ALSHEHRI et al., 2020).

Entretanto, a idade avançada também é um importante fator de risco ao acúmulo excessivo de gordura. Diante disto, a presente revisão evidenciou que as prevalências de sobrepeso/obesidade oscilaram entre 34,2\% (TAVARES et al., 2015) e 77,4\% (FERREIRA; MONTEIRO; SIMÕES, 2018), possivelmente, como algumas das consequências do aumento no armazenamento do tecido adiposo, que ocorre principalmente após os 40 anos (SANTOS et al., 2013).

No que se refere ao panorama epidemiológico da HAS, verificou-se que as prevalências para este desfecho se mostraram elevadas em todos os estudos analisados. O menor valor identificado foi na ordem de 62,8\% no estudo de Ferreira, Monteiro e Simões (2018), enquanto a maior prevalência foi observada no estudo de Turi et al. (2013), em que 76,8\% dos idosos apresentavam HAS.

Estes resultados remetem uma preocupante conjuntura, haja vista que os níveis pressóricos elevados podem gerar consideráveis lesões em órgãos de suma importância à vida, a exemplo do coração, cérebro e rins (MALACHIAS et al., 2016). Assim, em relação à mortalidade, estima-se que a HAS contribua para $50 \%$ das mortes por doenças cardiovasculares, $40 \%$ dos óbitos por acidente vascular cerebral e em 50\% dos casos de insuficiência renal (PARANÁ, 2018).

Ademais, observou-se no estudo de Ferreira Monteiro e Simões (2018), realizado com dados de 720 idosos, obtidos por meio do sistema de Vigilância de Fatores de Risco e Proteção para Doenças Crônicas por Inquérito Telefônico, que os avaliados com sobrepeso e os com obesidade apresentaram 1,7 e 3,85 vezes mais chances, respectivamente, para o acometimento da HAS quando comparados aos com peso adequado.

Por sua vez, Turi et al. (2013), averiguaram em um estudo realizado com 963 pessoas (65,0 \pm 9,0 anos) atendidas em cinco unidades básicas de saúde de Baurú-SP, nas quais os pacientes com excesso de peso e os com obesidade apresentaram, respectivamente, 1,2 e 1,4 maior prevalência de HAS, quando comparados aos eutróficos. 
De forma semelhante, Tavares et al. (2018) verificaram em um estudo realizado com 562 idosos, residentes na zona rural de Uberaba-MG, que os com a condição de sobrepeso/obesidade apresentaram 2,52 vezes mais chances para elevados níveis pressóricos.

Não obstante, Leal Neto, Barbosa e Meneghinias (2016) observaram em um estudo de base populacional, realizado com 477 indivíduos com idade $\geq 60$ anos, que as idosas e os idosos hipertensos possuíam, respectivamente, em média, um índice de massa corporal 3,22 e $2,27 \mathrm{~kg} / \mathrm{m}^{2}$ maior, quando comparados os avaliados de igual sexo, não hipertensos.

As associações identificadas entre o excesso de peso e a obesidade com a HAS pode ser explicada por alterações hemodinâmicas, como o aumento do débito cardíaco e uma maior resistência vascular periférica, além de desequilíbrios neuroendócrinos, ocasionados, principalmente, pelo acúmulo excessivo de gordura, sobretudo, na região visceral (KOLIAKI; LIATIS; KOKKINOS, 2019).

Além do mais, os mecanismos desta fisiopatologia, em pessoas com sobrepeso/obesidade, são decorrentes de uma maior estimulação ao Sistema Nervoso Simpático (SNS), feita por hormônios como a insulina e a leptina, bem como por uma constante ativação do sistema renina-angiotensina-aldosterona, devido à liberação de renina estimulada por meio do SNS e pelo angiotensinogênio, produzido pelo próprio tecido adiposo (LANDSBERG et al., 2013).

Entre as limitações verificadas nos estudos selecionados, destaca-se que todos utilizaram apenas o IMC para avaliação do estado nutricional, tendo em vista que este indicador antropométrico remete apenas a distribuição da massa corporal, em relação a estatura, e não os percentuais ou valores absolutos da massa gorda e massa livre de gordura.

Além disso, ainda não existe um consenso sobre os valores de referência, do referido indicador antropométrico, para determinação do excesso de peso e obesidade na população estudada, o que resultou na utilização de diferentes critérios para sua categorização.

\section{Conclusão}

Os resultados da presente revisão mostraram elevadas prevalências de sobrepeso/obesidade e hipertensão arterial sistêmica, bem como associações entre estas 
condições nos idosos avaliados.

Apesar de todos os estudos terem apresentado delineamento transversal, o que impossibilita a inferência de causa e efeito ou temporalidade entre as respectivas variáveis, as associações observadas evidenciam o excesso de peso e a obesidade com possíveis condições de vulnerabilidade para elevados níveis pressóricos.

Desta forma, torna-se imprescindível a adoção de ações de vigilância à saúde, a exemplo do monitoramento do estado nutricional, por meio de avaliações de indicadores antropométricos e a estimativa da composição corporal, a fim de triar os idosos com maior probabilidade de desenvolver a HAS.

\section{Referências}

BRASIL. Vigitel Brasil. (2019) Saúde Suplementar: vigilância de fatores de risco e proteção para doenças crônicas por inquérito telefônico. Estimativas sobre frequência e distribuição sociodemográfica de fatores de risco e proteção para doenças crônicas nas capitais dos 26 estados brasileiros e no Distrito Federal em 2018.

Calixto, I. T.; Prazeres, T. C. M. M. (2019). Uso da testosterona no envelhecimento masculino. Revista de Investigação Biomédica, v. 10, n. 3, p. 227-236.

Ferreira, C. C. D.; Monteiro, G. T. R.; Simões, T. C. (2018). Estado nutricional e fatores associados em idosos: evidências com base em inquérito telefônico. Revista Brasileira em Promoção da Saúde, v. 31, n. 1, p. 1-11.

Jafarinasabian, P. et al. (2017). Aging human body: changes in bone, muscle and body fat with consequent changes in nutrient intake. Journal of Endocrinology, p. JOE-16-0603.

Karvonen-Gutierrez, C.; Kim, C. (2016). Association of mid-life changes in body size, body composition and obesity status with the menopausal transition. In: Healthcare. Multidisciplinary Digital Publishing Institute.

Koliaki, C.; Liatis, S.; Kokkinos, A. (2019). Obesity and cardiovascular disease: revisiting an old relationship. Metabolism, v. 92, p. 98-107.

Landsberg, L. et al. 92023). Obesity-related hypertension: pathogenesis, cardiovascular risk, and treatment: a position paper of the Obesity Society and the American Society of Hypertension. The Journal of Clinical Hypertension, v. 15, n. 1, p. 14-33. 
Leal Neto, J. S.; Barbosa, A. R.; Meneghini, V. Diseases and chronic health conditions, multimorbidity and body mass index in older adults. Revista Brasileira de Cineantropometria \& Desempenho Humano, v. 18, n. 5, p. 509-519, 2016.

Lipschitz, D. A. (1994). Screening for nutritional status in the elderly. Primary Care, v. 21, n. 1 , p. 55-67.

Malachias, M. V. B. et al. (2016). VII Diretriz Brasileira de Hipertensão Arterial: Capítulo 1-Conceituação, Epidemiologia e Prevenção Primária. Arquivos Brasileiros de Cardiologia, v. 107, n. 3, p. 1-6.

Melnyk, B. M. et al. (2010). Evidence-based practice: step by step: the seven steps of evidence-based practice. AJN The American Journal of Nursing, v. 110, n. 1, p. 51-53.

Secretaria de Estado da Saúde do Paraná. (2018). Secretaria de Estado da Saúde do Paraná. Superintendência de Atenção à Saúde. Linha guia de hipertensão arterial / SAS. $2^{a}$ ed. Curitiba: SESA.

Souza, M. T.; Silva, M. D.; Carvalho, R. (2010). Revisão integrativa: o que é e como fazer. Einstein, v. 8, n. 1, p. 102-106, 2010.

Tavares, D. M. S. et al. (2028). Excesso de peso em idosos rurais: associação com as condições de saúde e qualidade de vida. Ciência \& Saúde Coletiva, v. 23, p. 913-922.

Turi, B. C. et al. (2013). Frequência de ocorrência e fatores associados à hipertensão arterial em pacientes do Sistema Único de Saúde. Revista Brasileira de Atividade Física \& Saúde, v. 18, n. 1, p. 43-52.

World Health Organization et al. (1998). Obesity: preventing and managing the global epidemic: report of a WHO consultation on obesity, Geneva, 3-5 June 1997. Geneva: World Health Organization. 